\title{
CONVERGENCE OF RELAXED TWO-STAGE MULTISPLITTING METHOD USING AN OUTER SPLITTING
}

\author{
JAE HEON Yun
}

\begin{abstract}
In this paper, we study the convergence of relaxed two-stage multisplitting method using $H$-compatible splittings or SOR multisplitting as inner splittings and an outer splitting for solving a linear system whose coefficient matrix is an $H$-matrix. We also provide numerical experiments for the convergence of the relaxed two-stage multisplitting method.
\end{abstract}

\section{Introduction}

In this paper, we consider relaxed two-stage multisplitting method for solving a linear system of the form

$$
A x=b, \quad x, b \in \mathbb{R}^{n},
$$

where $A \in \mathbb{R}^{n \times n}$ is a large sparse $H$-matrix. Multisplitting method was introduced by O'Leary and White [5] and was further studied by many authors $[3,4,6,8,9]$. The multisplitting method can be thought of as an extension and parallel generalization of the classical block Jacobi method [2].

A matrix $A=\left(a_{i j}\right) \in \mathbb{R}^{n \times n}$ is called monotone if $A^{-1} \geq 0$. A matrix $A=\left(a_{i j}\right) \in \mathbb{R}^{n \times n}$ is called an $M$-matrix if $A$ is monotone and $a_{i j} \leq 0$ for $i \neq j$. The comparison matrix $\langle A\rangle=\left(\alpha_{i j}\right)$ of a matrix $A=\left(a_{i j}\right)$ is defined by

$$
\alpha_{i j}=\left\{\begin{aligned}
\left|a_{i j}\right| & \text { if } i=j \\
-\left|a_{i j}\right| & \text { if } i \neq j .
\end{aligned}\right.
$$

A matrix $A$ is called an $H$-matrix if $\langle A\rangle$ is an $M$-matrix. A representation $A=M-N$ is called a splitting of $A$ when $M$ is nonsingular. A splitting $A=M-N$ is called regular if $M^{-1} \geq 0$ and $N \geq 0$, weak regular if $M^{-1} \geq 0$ and $M^{-1} N \geq 0, M$-splitting of $A$ if $M$ is an $M$-matrix and $N \geq 0$, and $H$ compatible splitting of $A$ if $\langle A\rangle=\langle M\rangle-|N|$.

Received February 9, 2009; Revised April 8, 2009.

2000 Mathematics Subject Classification. Primary 65F10, 65F15.

Key words and phrases. $H$-compatible splitting, SOR multisplitting, relaxed two-stage multisplitting method, $H$-matrix. 
A collection of triples $\left(M_{k}, N_{k}, E_{k}\right), k=1,2, \ldots, \ell$, is called a multisplitting of $A$ if $A=M_{k}-N_{k}$ is a splitting of $A$ for $k=1,2, \ldots, \ell$, and $E_{k}$ 's, called weighting matrices, are nonnegative diagonal matrices such that $\sum_{k=1}^{\ell} E_{k}=I$. The relaxed two-stage multisplitting method with a relaxation parameter $\beta>0$ using $A=M_{k}-N_{k}$ as outer splittings and $M_{k}=B_{k}-C_{k}$ as inner splittings is as follows.

Algorithm 1: Relaxed Two-stage Multisplitting method

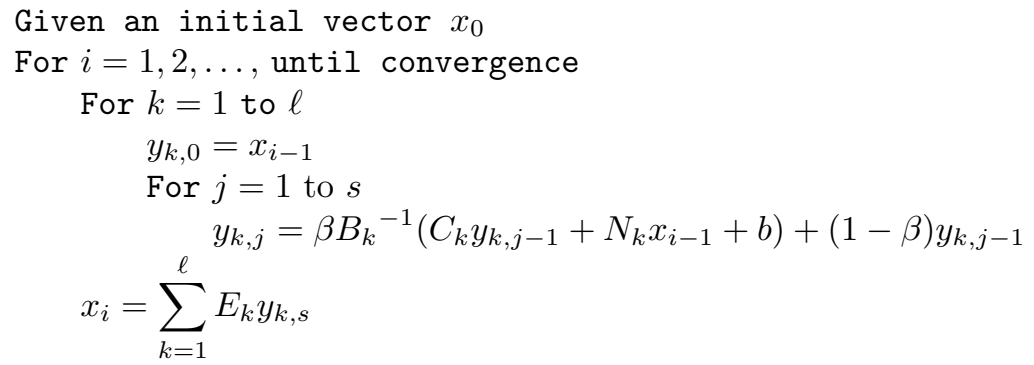

In Algorithm 1, it is assumed to be $s \geq 1$. Bru et al. [2] showed that if $0<\beta \leq 1$, then Algorithm 1 converges for an $H$-matrix $A$ under the assumption that both the outer splittings $A=M_{k}-N_{k}$ and the inner splittings $M_{k}=$ $B_{k}-C_{k}$ are $H$-compatible splittings.

In 1991, Wang [8] studied the convergence of relaxed multisplitting method associated with AOR multisplitting for solving the linear system (1). In this paper, we study the convergence of relaxed two-stage multisplitting method using $H$-compatible splittings or SOR multisplitting as inner splittings and an outer splitting for solving the linear system (1). This paper is organized as follows. In Section 2, we present some notation and well-known results. In Section 3, we provide convergence results of relaxed two-stage multisplitting method using $H$-compatible splittings or SOR multisplitting as inner splittings and an outer splitting. In Section 4, we also provide numerical experiments for the convergence of the relaxed two-stage multisplitting method.

\section{Preliminaries}

For a vector $x \in \mathbb{R}^{n}, x \geq 0(x>0)$ denotes that all components of $x$ are nonnegative (positive). For two vectors $x, y \in \mathbb{R}^{n}, x \geq y(x>y)$ means that $x-y \geq 0(x-y>0)$. For a vector $x \in \mathbb{R}^{n},|x|$ denotes the vector whose components are the absolute values of the corresponding components of $x$. These definitions carry immediately over to matrices. It follows that $|A| \geq 0$ for any matrix $A$ and $|A B| \leq|A||B|$ for any two matrices $A$ and $B$ of compatible size. Let $\operatorname{diag}(A)$ denote a diagonal matrix whose diagonal part coincides with the diagonal part of $A$, and let $\rho(A)$ denote the spectral radius of a square matrix $A$. Varga [7] showed that for any square matrices $A$ and $B$, $|A| \leq B$ implies $\rho(A) \leq \rho(B)$. It is well-known that if $A=M-N$ is a weak 
regular splitting, then $A^{-1} \geq 0$ if and only if $\rho\left(M^{-1} N\right)<1[1,7]$. It was shown that if $A \geq 0$ and there exists a vector $x>0$ and an $\alpha \geq 0$ such that $A x \leq \alpha x$, then $\rho(A) \leq \alpha[1]$. Frommer and Mayer [3] showed that $\left|A^{-1}\right| \leq\langle A\rangle^{-1}$ when $A$ is an $H$-matrix.

Theorem 2.1 ([2]). Let $A \in \mathbb{R}^{n \times n}$ be a monotone matrix. Assume that the outer splittings $A=M_{k}-N_{k}$ are regular and the inner splittings $M_{k}=B_{k}-C_{k}$ are weak regular. If $0<\beta \leq 1$, then the relaxed two-stage multisplitting method converges to the exact solution of $A x=b$ for any initial vector $x_{0}$.

Theorem $2.2([2])$. Let $A \in \mathbb{R}^{n \times n}$ be an H-matrix. Assume that the outer splittings $A=M_{k}-N_{k}$ are $H$-compatible splittings and the inner splittings $M_{k}=B_{k}-C_{k}$ are $H$-compatible splittings. If $0<\beta \leq 1$, then the relaxed two-stage multisplitting method converges to the exact solution of $A x=b$ for any initial vector $x_{0}$.

The SOR multisplitting to be used in this paper is defined as follows.

Definition 1. Let $0<\omega<2$ and $A=D-L_{k}-U_{k}$ for $k=1,2, \ldots, \ell$, where $D=\operatorname{diag}(A), L_{k}$ 's are strictly lower triangular matrices, and $U_{k}$ 's are general matrices. $\left(M_{k}(\omega), N_{k}(\omega), E_{k}\right), k=1,2, \ldots, \ell$, is called the SOR multisplitting of $A$ if $\left(M_{k}(\omega), N_{k}(\omega), E_{k}\right), k=1,2, \ldots, \ell$, is a multisplitting of $A, M_{k}(\omega)=$ $\frac{1}{\omega}\left(D-\omega L_{k}\right)$, and $N_{k}(\omega)=\frac{1}{\omega}\left((1-\omega) D+\omega U_{k}\right)$.

If $\omega=1$ in Definition 1, then the SOR multisplitting of $A$ is called the GaussSeidel multisplitting of $A$. In this case, $M_{k}(\omega)=D-L_{k}$ and $N_{k}(\omega)=U_{k}$.

\section{Convergence results of relaxed two-stage multisplitting method}

In this section, we consider convergence of relaxed two-stage multisplitting method (Algorithm 1) with a relaxation parameter $\beta>0$ using an outer splitting $A=M-N$ and inner splittings $M=B_{k}-C_{k}$. Then, Algorithm 1 can be written as $x_{i}=H_{\beta} x_{i-1}+P_{\beta} b, i=1,2, \ldots$, where

$$
\begin{aligned}
& H_{\beta}=\sum_{k=1}^{\ell} E_{k}\left(R_{\beta, k}\right)^{s}+\beta \sum_{k=1}^{\ell} E_{k}\left(\sum_{j=0}^{s-1}\left(R_{\beta, k}\right)^{j}\right) B_{k}^{-1} N, \\
& P_{\beta}=\beta \sum_{k=1}^{\ell} E_{k}\left(\sum_{j=0}^{s-1}\left(R_{\beta, k}\right)^{j}\right) B_{k}^{-1},
\end{aligned}
$$

where $R_{\beta, k}=\beta B_{k}^{-1} C_{k}+(1-\beta) I$. The $H_{\beta}$ is called an iteration matrix for the relaxed two-stage multisplitting method with a relaxation parameter $\beta>0$ and $s$ inner iterations. Then, it can be shown that $P_{\beta} A=I-H_{\beta}$ and the relaxed two-stage multisplitting method with a relaxation parameter $\beta>0$ converges to the exact solution of $A x=b$ for any initial vector $x_{0}$ if and only if $\rho\left(H_{\beta}\right)<1$. First, we provide convergence result of the relaxed two-stage multisplitting method using $H$-compatible splittings. 
Theorem 3.1. Let $A \in \mathbb{R}^{n \times n}$ be an $H$-matrix and $A=M-N$ be an $H$ compatible splitting of $A$. Let $M=B_{k}-C_{k}$ be an $H$-compatible splitting such that $\operatorname{diag}\left(B_{k}\right)=\operatorname{diag}(M)=D$ for each $1 \leq k \leq \ell$, and let $B=D-M$. Then, the relaxed two-stage multisplitting method using an outer splitting $A=$ $M-N$ and inner splittings $M=B_{k}-C_{k}, k=1,2, \ldots, \ell$, converges to the exact solution of $A x=b$ for any initial vector $x_{0}$ if $0<\beta<\frac{2}{1+\alpha}$, where $\alpha=\rho\left(|D|^{-1}(|B|+|N|)\right)$.

Proof. For $0<\beta \leq 1$, this theorem follows directly from Theorem 2.2. Now we consider the case of $1<\beta<\frac{2}{1+\alpha}$. Let

$$
\begin{gathered}
H_{\beta}=\sum_{k=1}^{\ell} E_{k} H_{\beta, k}, \quad H_{\beta, k}=\left(R_{\beta, k}\right)^{s}+\beta \sum_{j=0}^{s-1}\left(R_{\beta, k}\right)^{j} B_{k}^{-1} N, \\
\tilde{H}_{\beta}=\sum_{k=1}^{\ell} E_{k} \tilde{H}_{\beta, k}, \quad \tilde{H}_{\beta, k}=\left(\tilde{R}_{\beta, k}\right)^{s}+\beta \sum_{j=0}^{s-1}\left(\tilde{R}_{\beta, k}\right)^{j}\left\langle B_{k}\right\rangle^{-1}|N|,
\end{gathered}
$$

where $R_{\beta, k}=\beta B_{k}^{-1} C_{k}+(1-\beta) I$ and $\tilde{R}_{\beta, k}=\beta\left\langle B_{k}\right\rangle^{-1}\left|C_{k}\right|+(\beta-1) I$. Let

$$
\tilde{A}_{\beta, k}=\frac{2-\beta}{\beta}\left\langle B_{k}\right\rangle-\left|C_{k}\right|-|N| \text { and } \tilde{A}_{\beta}=\frac{2-\beta}{\beta}|D|-|B|-|N| \text {. }
$$

Notice that $M$ and $B_{k}$ are $H$-matrices since $A=M-N$ and $M=B_{k}-C_{k}$ are $H$-compatible splittings. Since it can be easily shown that $\left|R_{\beta, k}\right| \leq \tilde{R}_{\beta, k}$, $\left|H_{\beta, k}\right| \leq \tilde{H}_{\beta, k}$ and thus

$$
\left|H_{\beta}\right| \leq \tilde{H}_{\beta}
$$

Since $\operatorname{diag}\left(B_{k}\right)=\operatorname{diag}(M)=D,\langle M\rangle=|D|-|B|$ and $\left\langle B_{k}\right\rangle \leq|D|$. Since $\langle A\rangle=|D|-(|B|+|N|)=\left\langle B_{k}\right\rangle-\left(\left|C_{k}\right|+|N|\right)$ are regular splittings of $\langle A\rangle$ and $\left\langle B_{k}\right\rangle^{-1} \geq|D|^{-1}, \rho\left(\left\langle B_{k}\right\rangle^{-1}\left(\left|C_{k}\right|+|N|\right)\right) \leq \alpha<1$. Since $\beta<\frac{2}{1+\alpha}, \frac{\beta \alpha}{2-\beta}<1$ and thus $I-\tilde{R}_{\beta, k}=(2-\beta) I-\beta\left\langle B_{k}\right\rangle^{-1}\left|C_{k}\right|$ is nonsingular. Hence, one obtains

$$
\begin{aligned}
\tilde{H}_{\beta, k} & =\left(\tilde{R}_{\beta, k}\right)^{s}+\beta\left(I-\left(\tilde{R}_{\beta, k}\right)^{s}\right)\left(I-\tilde{R}_{\beta, k}\right)^{-1}\left\langle B_{k}\right\rangle^{-1}|N| \\
& =I-\left(I-\left(\tilde{R}_{\beta, k}\right)^{s}\right)\left(I-\beta\left(I-\tilde{R}_{\beta, k}\right)^{-1}\left\langle B_{k}\right\rangle^{-1}|N|\right) \\
& =I-\left(I-\left(\tilde{R}_{\beta, k}\right)^{s}\right)\left(I-\tilde{R}_{\beta, k}\right)^{-1}\left(I-\tilde{R}_{\beta, k}-\beta\left\langle B_{k}\right\rangle^{-1}|N|\right) \\
& =I-\sum_{j=0}^{s-1}\left(\tilde{R}_{\beta, k}\right)^{j}\left((2-\beta) I-\beta\left\langle B_{k}\right\rangle^{-1}\left|C_{k}\right|-\beta\left\langle B_{k}\right\rangle^{-1}|N|\right) \\
& =I-\beta \sum_{j=0}^{s-1}\left(\tilde{R}_{\beta, k}\right)^{j}\left\langle B_{k}\right\rangle^{-1}\left(\frac{2-\beta}{\beta}\left\langle B_{k}\right\rangle-\left|C_{k}\right|-|N|\right) \\
& =I-\beta \sum_{j=0}^{s-1}\left(\tilde{R}_{\beta, k}\right)^{j}\left\langle B_{k}\right\rangle^{-1} \tilde{A}_{\beta, k} .
\end{aligned}
$$


Let $E_{k}=D-B_{k}$ for each $k$. Then $\left\langle B_{k}\right\rangle=|D|-\left|E_{k}\right|$ and $|B|=\left|E_{k}\right|+\left|C_{k}\right|$. Since $1<\beta<\frac{2}{1+\alpha}$, one obtains

$$
\begin{aligned}
\tilde{A}_{\beta, k} & =\frac{2-\beta}{\beta}\left\langle B_{k}\right\rangle-\left|C_{k}\right|-|N| \\
& =\frac{2-\beta}{\beta}|D|-\frac{2-\beta}{\beta}\left|E_{k}\right|-\left|C_{k}\right|-|N| \\
& \geq \frac{2-\beta}{\beta}|D|-\left|E_{k}\right|-\left|C_{k}\right|-|N| \\
& =\frac{2-\beta}{\beta}|D|-|B|-|N| \\
& =\tilde{A}_{\beta}
\end{aligned}
$$

for each $k$. Since $\tilde{A}_{\beta}=\frac{2-\beta}{\beta}|D|-(|B|+|N|)$ is a regular splitting of $\tilde{A}_{\beta}$ and $\frac{\beta \alpha}{2-\beta}<1, \tilde{A}_{\beta}^{-1} \geq 0$. Since $\tilde{R}_{\beta, k}$ and $\left\langle B_{k}\right\rangle^{-1}$ are nonnegative, from (3) and (4) one obtains

$$
\tilde{H}_{\beta, k} \leq I-\beta \sum_{j=0}^{s-1}\left(\tilde{R}_{\beta, k}\right)^{j}\left\langle B_{k}\right\rangle^{-1} \tilde{A}_{\beta} .
$$

Let $e=(1,1, \ldots, 1)^{T}$ and $v=\tilde{A}_{\beta}^{-1} e$. Then $v>0$ and $\left\langle B_{k}\right\rangle^{-1} e>0$. Using these relations and (5),

$$
\tilde{H}_{\beta, k} v \leq v-\beta \sum_{j=0}^{s-1}\left(\tilde{R}_{\beta, k}\right)^{j}\left\langle B_{k}\right\rangle^{-1} e \leq v-\beta\left\langle B_{k}\right\rangle^{-1} e<v .
$$

From (6), there exists $\theta_{\beta, k} \in[0,1)$ such that

$$
\tilde{H}_{\beta, k} v \leq \theta_{\beta, k} v
$$

for each $k$. Let $\theta_{\beta}=\max \left\{\theta_{\beta, k} \mid 1 \leq k \leq \ell\right\}$. It is clear that $\theta_{\beta}<1$. From (2) and (7),

$$
\left|H_{\beta}\right| v \leq \tilde{H}_{\beta} v=\sum_{k=1}^{\ell} E_{k} \tilde{H}_{\beta, k} v \leq \sum_{k=1}^{\ell} \theta_{\beta, k} E_{k} v \leq \theta_{\beta} v .
$$

From (8), $\rho\left(\left|H_{\beta}\right|\right) \leq \theta_{\beta}<1$ and hence $\rho\left(H_{\beta}\right) \leq \theta_{\beta}<1$ for $1<\beta<\frac{2}{1+\alpha}$. Therefore, the proof is complete.

In Theorem 3.1, notice that $\frac{2}{1+\alpha}>1$ since $\alpha<1$. It means that Theorem 3.1 can be viewed as an extension of Theorem 2.2. The following corollary for an $M$-matrix $A$ is directly obtained from Theorem 3.1

Corollary 3.2. Let $A \in \mathbb{R}^{n \times n}$ be an $M$-matrix and $A=M-N$ be an $M$ splitting of $A$. Let $M=B_{k}-C_{k}$ be an $M$-splitting such that $\operatorname{diag}\left(B_{k}\right)=$ $\operatorname{diag}(M)=D$ for each $1 \leq k \leq \ell$, and let $B=D-M$. Then, the relaxed two-stage multisplitting method using an outer splitting $A=M-N$ and inner 
splittings $M=B_{k}-C_{k}, k=1,2, \ldots, \ell$, converges to the exact solution of $A x=b$ for any initial vector $x_{0}$ if $0<\beta<\frac{2}{1+\alpha}$, where $\alpha=\rho\left(D^{-1}(B+N)\right)$.

We next provide convergence results of the relaxed two-stage multisplitting method using SOR multisplitting.

Theorem 3.3. Let $A \in \mathbb{R}^{n \times n}$ be an $H$-matrix and $A=M-N$ be an $H$ compatible splitting of $A$. Let $M=D-B=D-L_{k}-U_{k}(1 \leq k \leq \ell)$ such that $\langle M\rangle=|D|-\left|L_{k}\right|-\left|U_{k}\right|$, where $D=\operatorname{diag}(M), L_{k}$ is a strictly lower triangular matrix, and $U_{k}$ is a general matrix, and let $\left(B_{k}(\omega), C_{k}(\omega), E_{k}\right), k=1,2, \ldots, \ell$, be the SOR multisplitting of $M$. Let $\alpha=\max \left\{\max \left\{\rho\left(\left\langle B_{k}(\omega)\right\rangle^{-1}\left|C_{k}(\omega)\right|\right) \mid 1 \leq\right.\right.$ $\left.k \leq \ell\}, \rho\left(|D|^{-1}(|B|+|N|)\right)\right\}$. Then, the relaxed two-stage multisplitting method using an outer splitting $A=M-N$ and inner splittings $M=B_{k}(\omega)-C_{k}(\omega)$, $k=1,2, \ldots, \ell$, converges to the exact solution of $A x=b$ for any initial vector $x_{0}$ if $0<\omega \leq 1$ and $0<\beta<\frac{2}{2-\omega(1-\alpha)}$.

Proof. Notice that $\left\langle B_{k}(\omega)\right\rangle=\frac{1}{\omega}\left(|D|-\omega\left|L_{k}\right|\right)$ and $\left|C_{k}(\omega)\right|=\frac{1}{\omega}((1-\omega)|D|+$ $\left.\omega\left|U_{k}\right|\right)$ for $0<\omega \leq 1$. It follows that $M=B_{k}(\omega)-C_{k}(\omega)$ is an $H$-compatible splitting of $M$, that is, $\langle M\rangle=\left\langle B_{k}(\omega)\right\rangle-\left|C_{k}(\omega)\right|$. Since $\langle A\rangle=|D|-(|B|+|N|)$ and $\langle M\rangle=\left\langle B_{k}(\omega)\right\rangle-\left|C_{k}(\omega)\right|$ are regular splittings, it is clear that $\alpha<1$. For $0<\omega \leq 1$ and $0<\beta \leq 1$, this theorem follows directly from Theorem 2.2 since both $A=M-N$ and $M=B_{k}(\omega)-C_{k}(\omega)$ are $H$-compatible splittings. Now we consider the case of $0<\omega \leq 1$ and $1<\beta<\frac{2}{2-\omega(1-\alpha)}$. Let

$$
\begin{gathered}
R_{\beta, \omega, k}=\beta\left(B_{k}(\omega)\right)^{-1} C_{k}(\omega)+(1-\beta) I, \\
\tilde{R}_{\beta, \omega, k}=\beta\left\langle B_{k}(\omega)\right\rangle^{-1}\left|C_{k}(\omega)\right|+(\beta-1) I, \\
H_{\beta, \omega}=\sum_{k=1}^{\ell} E_{k} H_{\beta, \omega, k}, \quad H_{\beta, \omega, k}=\left(R_{\beta, \omega, k}\right)^{s}+\beta \sum_{j=0}^{s-1}\left(R_{\beta, \omega, k}\right)^{j}\left(B_{k}(\omega)\right)^{-1} N, \\
\tilde{H}_{\beta, \omega}=\sum_{k=1}^{\ell} E_{k} \tilde{H}_{\beta, \omega, k}, \quad \tilde{H}_{\beta, \omega, k}=\left(\tilde{R}_{\beta, \omega, k}\right)^{s}+\beta \sum_{j=0}^{s-1}\left(\tilde{R}_{\beta, \omega, k}\right)^{j}\left\langle B_{k}(\omega)\right\rangle^{-1}|N| .
\end{gathered}
$$

Let

$\tilde{A}_{\beta, \omega, k}=\frac{2-\beta}{\beta}\left\langle B_{k}(\omega)\right\rangle-\left|C_{k}(\omega)\right|-|N|$ and $\tilde{A}_{\beta, \omega}=\frac{2-2 \beta+\beta \omega}{\beta \omega}|D|-|B|-|N|$.

Since $B_{k}(\omega)$ is an $H$-matrix, $\left|\left(B_{k}(\omega)\right)^{-1}\right| \leq\left\langle B_{k}(\omega)\right\rangle^{-1}$ and thus $\left|R_{\beta, \omega, k}\right| \leq$ $\tilde{R}_{\beta, \omega, k}$. It follows that

$$
\left|H_{\beta, \omega}\right| \leq \tilde{H}_{\beta, \omega}
$$


Since $\beta<\frac{2}{2-\omega(1-\alpha)} \leq \frac{2}{1+\alpha}, \frac{\beta \alpha}{2-\beta}<1$ and thus $I-\tilde{R}_{\beta, \omega, k}=(2-\beta) I-$ $\beta\left\langle B_{k}(\omega)\right\rangle^{-1}\left|C_{k}(\omega)\right|$ is nonsingular. Hence, one obtains

$$
\begin{aligned}
\tilde{H}_{\beta, \omega, k} & =\left(\tilde{R}_{\beta, \omega, k}\right)^{s}+\beta\left(I-\left(\tilde{R}_{\beta, \omega, k}\right)^{s}\right)\left(I-\tilde{R}_{\beta, \omega, k}\right)^{-1}\left\langle B_{k}(\omega)\right\rangle^{-1}|N| \\
& =I-\left(I-\left(\tilde{R}_{\beta, \omega, k}\right)^{s}\right)\left(I-\beta\left(I-\tilde{R}_{\beta, \omega, k}\right)^{-1}\left\langle B_{k}(\omega)\right\rangle^{-1}|N|\right) \\
& =I-\left(I-\left(\tilde{R}_{\beta, \omega, k}\right)^{s}\right)\left(I-\tilde{R}_{\beta, \omega, k}\right)^{-1}\left(I-\tilde{R}_{\beta, \omega, k}-\beta\left\langle B_{k}(\omega)\right\rangle^{-1}|N|\right) \\
& =I-\sum_{j=0}^{s-1}\left(\tilde{R}_{\beta, \omega, k}\right)^{j}\left((2-\beta) I-\beta\left\langle B_{k}(\omega)\right\rangle^{-1}\left|C_{k}(\omega)\right|-\beta\left\langle B_{k}(\omega)\right\rangle^{-1}|N|\right) \\
& =I-\beta \sum_{j=0}^{s-1}\left(\tilde{R}_{\beta, \omega, k}\right)^{j}\left\langle B_{k}(\omega)\right\rangle^{-1}\left(\frac{2-\beta}{\beta}\left\langle B_{k}(\omega)\right\rangle-\left|C_{k}(\omega)\right|-|N|\right) \\
& =I-\beta \sum_{j=0}^{s-1}\left(\tilde{R}_{\beta, \omega, k}\right)^{j}\left\langle B_{k}(\omega)\right\rangle^{-1} \tilde{A}_{\beta, \omega, k} .
\end{aligned}
$$

Since $\beta>1$ and $|B|=\left|L_{k}\right|+\left|U_{k}\right|$ for every $k$, one obtains

$$
\begin{aligned}
\tilde{A}_{\beta, \omega, k} & =\frac{2-2 \beta+\beta \omega}{\beta \omega}|D|-\frac{2-\beta}{\beta}\left|L_{k}\right|-\left|U_{k}\right|-|N| \\
& \geq \frac{2-2 \beta+\beta \omega}{\beta \omega}|D|-\left|L_{k}\right|-\left|U_{k}\right|-|N| \\
& =\frac{2-2 \beta+\beta \omega}{\beta \omega}|D|-|B|-|N| \\
& =\tilde{A}_{\beta, \omega}
\end{aligned}
$$

for each $k$. Since $\beta<\frac{2}{2-\omega(1-\alpha)}, 2-2 \beta+\beta \omega>0$ and $\frac{\beta \omega \alpha}{2-2 \beta+\beta \omega}<1$. It follows that $\tilde{A}_{\beta, \omega}=\frac{2-2 \beta+\beta \omega}{\beta \omega}|D|-(|B|+|N|)$ is a regular splitting of $\tilde{A}_{\beta, \omega}$ and thus $\tilde{A}_{\beta, \omega}^{-1} \geq 0$. Since $\tilde{R}_{\beta, \omega, k}$ and $\left\langle B_{k}(\omega)\right\rangle^{-1}$ are nonnegative, from (10) and (11) one obtains

$$
\tilde{H}_{\beta, \omega, k} \leq I-\beta \sum_{j=0}^{s-1}\left(\tilde{R}_{\beta, \omega, k}\right)^{j}\left\langle B_{k}(\omega)\right\rangle^{-1} \tilde{A}_{\beta, \omega}
$$

Let $e=(1,1, \ldots, 1)^{T}$ and $v=\tilde{A}_{\beta, \omega}^{-1} e$. Then $v>0$ and $\left\langle B_{k}(\omega)\right\rangle^{-1} e>0$. Using these relations and (12),

$$
\tilde{H}_{\beta, \omega, k} v \leq v-\beta \sum_{j=0}^{s-1}\left(\tilde{R}_{\beta, \omega, k}\right)^{j}\left\langle B_{k}(\omega)\right\rangle^{-1} e \leq v-\beta\left\langle B_{k}(\omega)\right\rangle^{-1} e<v .
$$

From (13), there exists a $\theta_{\beta, \omega, k} \in[0,1)$ such that

$$
\tilde{H}_{\beta, \omega, k} v \leq \theta_{\beta, \omega, k} v
$$


for each $k$. Let $\theta_{\beta, \omega}=\max \left\{\theta_{\beta, \omega, k} \mid 1 \leq k \leq \ell\right\}$. It is clear that $\theta_{\beta, \omega}<1$. From (9) and (14),

$$
\left|H_{\beta, \omega}\right| v \leq \tilde{H}_{\beta, \omega} v=\sum_{k=1}^{\ell} E_{k} \tilde{H}_{\beta, \omega, k} v \leq \sum_{k=1}^{\ell} \theta_{\beta, \omega, k} E_{k} v \leq \theta_{\beta, \omega} v .
$$

From (15), $\rho\left(\left|H_{\beta, \omega}\right|\right) \leq \theta_{\beta, \omega}<1$ and hence $\rho\left(H_{\beta, \omega}\right) \leq \theta_{\beta, \omega}<1$ for $0<\omega \leq 1$ and $1<\beta<\frac{2}{2-\omega(1-\alpha)}$. Therefore, the proof is complete.

Theorem 3.4. Let $A \in \mathbb{R}^{n \times n}$ be an $H$-matrix and $A=M-N$ be an $H$ compatible splitting of $A$. Let $M=D-B=D-L_{k}-U_{k}(1 \leq k \leq \ell)$ such that $\langle M\rangle=|D|-\left|L_{k}\right|-\left|U_{k}\right|$, where $D=\operatorname{diag}(M), L_{k}$ is a strictly lower triangular matrix, and $U_{k}$ is a general matrix, and let $\left(B_{k}(\omega), C_{k}(\omega), E_{k}\right)$, $k=1,2, \ldots, \ell$, be the SOR multisplitting of $M$. Let $\delta=\rho\left(|D|^{-1}(|B|+|N|)\right)$ and $\alpha=\max \left\{\delta, \max \left\{\rho\left(\left\langle B_{k}(\omega)\right\rangle^{-1}\left|C_{k}(\omega)\right|\right) \mid 1 \leq k \leq \ell\right\}\right\}$. Let

$$
H_{\beta, \omega}=\sum_{k=1}^{\ell} E_{k}\left(\left(R_{\beta, \omega, k}\right)^{s}+\beta \sum_{j=0}^{s-1}\left(R_{\beta, \omega, k}\right)^{j}\left(B_{k}(\omega)\right)^{-1} N\right)
$$

be an iteration matrix of the relaxed two-stage multisplitting method using an outer splitting $A=M-N$ and inner splittings $M=B_{k}(\omega)-C_{k}(\omega)$, where $R_{\beta, \omega, k}=\beta\left(B_{k}(\omega)\right)^{-1} C_{k}(\omega)+(1-\beta) I$. Then the following hold.

(a) If $1<\omega<\frac{2}{1+\delta}$ and $0<\beta \leq 1$, then $\rho\left(H_{\beta, \omega}\right)<1$.

(b) If $\omega>1$ is chosen so that $\omega(1+\alpha)<2$ and if $0<\beta<\frac{2}{\omega(1+\alpha)}$, then $\rho\left(H_{\beta, \omega}\right)<1$.

Proof. Let $\hat{M}=\left\langle B_{k}(\omega)\right\rangle-\left|C_{k}(\omega)\right|$ for $1<\omega<\frac{2}{1+\delta}$. Since $\left\langle B_{k}(\omega)\right\rangle=$ $\frac{1}{\omega}\left(|D|-\omega\left|L_{k}\right|\right)$ and $\left|C_{k}(\omega)\right|=\frac{1}{\omega}\left((\omega-1)|D|+\omega\left|U_{k}\right|\right), \hat{M}=\left\langle B_{k}(\omega)\right\rangle-\left|C_{k}(\omega)\right|=$ $\frac{2-\omega}{\omega}|D|-|B|$ are regular splittings of $\hat{M}$. Since $\frac{\omega \delta}{2-\omega}<1, \hat{M}$ is an $M$-matrix and thus $\alpha<1$. Let

$$
\begin{gathered}
\hat{R}_{\beta, \omega, k}=\beta\left\langle B_{k}(\omega)\right\rangle^{-1}\left|C_{k}(\omega)\right|+(1-\beta) I, \\
\tilde{R}_{\beta, \omega, k}=\beta\left\langle B_{k}(\omega)\right\rangle^{-1}\left|C_{k}(\omega)\right|+(\beta-1) I, \\
\hat{H}_{\beta, \omega}=\sum_{k=1}^{\ell} E_{k} \hat{H}_{\beta, \omega, k}, \quad \hat{H}_{\beta, \omega, k}=\left(\hat{R}_{\beta, \omega, k}\right)^{s}+\beta \sum_{j=0}^{s-1}\left(\hat{R}_{\beta, \omega, k}\right)^{j}\left\langle B_{k}(\omega)\right\rangle^{-1}|N|, \\
\tilde{H}_{\beta, \omega}=\sum_{k=1}^{\ell} E_{k} \tilde{H}_{\beta, \omega, k}, \quad \tilde{H}_{\beta, \omega, k}=\left(\tilde{R}_{\beta, \omega, k}\right)^{s}+\beta \sum_{j=0}^{s-1}\left(\tilde{R}_{\beta, \omega, k}\right)^{j}\left\langle B_{k}(\omega)\right\rangle^{-1}|N| .
\end{gathered}
$$

We first prove part (a). Let $\hat{A}=\hat{M}-|N|$. Then $\hat{A}=\hat{M}-|N|=\frac{2-\omega}{\omega}|D|-$ $(|B|+|N|)$ are regular splittings of $\hat{A}$. Since $\frac{\omega \delta}{2-\omega}<1, \hat{A}$ is also an $M$-matrix. Since $\hat{H}_{\beta, \omega}$ can be viewed as an iteration matrix of the relaxed two-stage multisplitting method using an outer splitting $\hat{A}=\hat{M}-|N|$ and inner splittings $\hat{M}=\left\langle B_{k}(\omega)\right\rangle-\left|C_{k}(\omega)\right|, \rho\left(\hat{H}_{\beta, \omega}\right)<1$ is obtained from Theorem 2.1. Since it 
can be easily shown that $\left|H_{\beta, \omega}\right| \leq \hat{H}_{\beta, \omega}, \rho\left(H_{\beta, \omega}\right)<1$. Next we prove part (b). Assume that $\omega>1$ is chosen so that $\omega(1+\alpha)<2$. Then $\omega(1+\delta)<2$. For $0<\beta \leq 1, \rho\left(H_{\beta, \omega}\right)<1$ is directly obtained from part (a). Now we consider the case of $1<\beta<\frac{2}{\omega(1+\alpha)}$. Let

$$
\tilde{A}_{\beta, \omega, k}=\frac{2-\beta}{\beta}\left\langle B_{k}(\omega)\right\rangle-\left|C_{k}(\omega)\right|-|N| \text { and } \tilde{A}_{\beta, \omega}=\frac{2-\beta \omega}{\beta \omega}|D|-|B|-|N| .
$$

Since $B_{k}(\omega)$ is an $H$-matrix, $\left|R_{\beta, \omega, k}\right| \leq \tilde{R}_{\beta, \omega, k}$ and thus $\left|H_{\beta, \omega}\right| \leq \tilde{H}_{\beta, \omega}$. Since $\omega>1, \beta<\frac{2}{\omega(1+\alpha)}<\frac{2}{(1+\alpha)}$ and so $\frac{\beta \alpha}{2-\beta}<1$. It follows that $I-\tilde{R}_{\beta, \omega, k}=$ $(2-\beta) I-\beta\left\langle B_{k}(\omega)\right\rangle^{-1}\left|C_{k}(\omega)\right|$ is nonsingular. Hence, one obtains

$$
\tilde{H}_{\beta, \omega, k}=I-\beta \sum_{j=0}^{s-1}\left(\tilde{R}_{\beta, \omega, k}\right)^{j}\left\langle B_{k}(\omega)\right\rangle^{-1} \tilde{A}_{\beta, \omega, k} .
$$

Since $\beta>1$ and $|B|=\left|L_{k}\right|+\left|U_{k}\right|$ for every $k$, one obtains

$$
\begin{aligned}
\tilde{A}_{\beta, \omega, k} & =\frac{2-\beta \omega}{\beta \omega}|D|-\frac{2-\beta}{\beta}\left|L_{k}\right|-\left|U_{k}\right|-|N| \\
& \geq \frac{2-\beta \omega}{\beta \omega}|D|-\left|L_{k}\right|-\left|U_{k}\right|-|N| \\
& =\frac{2-\beta \omega}{\beta \omega}|D|-|B|-|N| \\
& =\tilde{A}_{\beta, \omega}
\end{aligned}
$$

for each $k$. Since $\beta<\frac{2}{\omega(1+\alpha)}, 2-\beta \omega>0$ and $\frac{\beta \omega \alpha}{2-\beta \omega}<1$. It follows that $\tilde{A}_{\beta, \omega}=\frac{2-\beta \omega}{\beta \omega}|D|-(|B|+|N|)$ is a regular splitting of $\tilde{A}_{\beta, \omega}$ and thus $\tilde{A}_{\beta, \omega}^{-1} \geq 0$. Since $\tilde{R}_{\beta, \omega, k}$ and $\left\langle B_{k}(\omega)\right\rangle^{-1}$ are nonnegative, from (16) and (17) one obtains

$$
\tilde{H}_{\beta, \omega, k} \leq I-\beta \sum_{j=0}^{s-1}\left(\tilde{R}_{\beta, \omega, k}\right)^{j}\left\langle B_{k}(\omega)\right\rangle^{-1} \tilde{A}_{\beta, \omega}
$$

Let $e=(1,1, \ldots, 1)^{T}$ and $v=\tilde{A}_{\beta, \omega}^{-1} e$. Then $v>0$ and $\left\langle B_{k}(\omega)\right\rangle^{-1} e>0$. Using these relations and (18),

$$
\tilde{H}_{\beta, \omega, k} v \leq v-\beta \sum_{j=0}^{s-1}\left(\tilde{R}_{\beta, \omega, k}\right)^{j}\left\langle B_{k}(\omega)\right\rangle^{-1} e \leq v-\beta\left\langle B_{k}(\omega)\right\rangle^{-1} e<v .
$$

The remaining part of the proof can be done in a similar way as was done in that of Theorem 3.3. Hence, $\rho\left(H_{\beta, \omega}\right)<1$ is obtained for $1<\beta<\frac{2}{\omega(1+\alpha)}$. Therefore, the proof is complete.

Notice that $1<\frac{2}{1+\alpha} \leq \frac{2}{1+\delta}$ in Theorem 3.4. The following theorem is directly obtained by combining Theorems 3.3 and 3.4 . 
Theorem 3.5. Let $A \in \mathbb{R}^{n \times n}$ be an $H$-matrix and $A=M-N$ be an $H$ compatible splitting of $A$. Let $M=D-B=D-L_{k}-U_{k}(1 \leq k \leq \ell)$ such that $\langle M\rangle=|D|-\left|L_{k}\right|-\left|U_{k}\right|$, where $D=\operatorname{diag}(M), L_{k}$ is a strictly lower triangular matrix, and $U_{k}$ is a general matrix, and let $\left(B_{k}(\omega), C_{k}(\omega), E_{k}\right)$, $k=1,2, \ldots, \ell$, be the SOR multisplitting of $M$. Let $\delta=\rho\left(|D|^{-1}(|B|+|N|)\right)$ and $\alpha=\max \left\{\delta, \max \left\{\rho\left(\left\langle B_{k}(\omega)\right\rangle^{-1}\left|C_{k}(\omega)\right|\right) \mid 1 \leq k \leq \ell\right\}\right\}$. Let

$$
H_{\beta, \omega}=\sum_{k=1}^{\ell} E_{k}\left(\left(R_{\beta, \omega, k}\right)^{s}+\beta \sum_{j=0}^{s-1}\left(R_{\beta, \omega, k}\right)^{j}\left(B_{k}(\omega)\right)^{-1} N\right)
$$

be an iteration matrix of the relaxed two-stage multisplitting method using an outer splitting $A=M-N$ and inner splittings $M=B_{k}(\omega)-C_{k}(\omega)$, where $R_{\beta, \omega, k}=\beta\left(B_{k}(\omega)\right)^{-1} C_{k}(\omega)+(1-\beta) I$. Then the following hold.

(a) If $1<\omega<\frac{2}{1+\delta}$ and $0<\beta \leq 1$, then $\rho\left(H_{\beta, \omega}\right)<1$.

(b) If $\omega>0$ is chosen so that $\omega(1+\alpha)<2$ and if $0<\beta<\frac{2}{1+\omega \alpha+|1-\omega|}$, then $\rho\left(H_{\beta, \omega}\right)<1$.

In Theorem 3.5, notice that if $0<\omega \leq 1$, then the condition $\omega(1+\alpha)<2$ in part (b) is automatically satisfied from the fact that $\alpha<1$. Also notice that the upper bound of $\beta$, which is $\frac{2}{1+\omega \alpha+|1-\omega|}$, is greater than 1 when $\omega(1+$ $\alpha)<2$. The following corollary for an $M$-matrix $A$ is directly obtained from Theorem 3.5.

Corollary 3.6. Let $A \in \mathbb{R}^{n \times n}$ be an $M$-matrix and $A=M-N$ be an $M$ splitting of $A$. Let $M=D-B=D-L_{k}-U_{k}(1 \leq k \leq \ell)$, where $D=\operatorname{diag}(M)$, $L_{k} \geq 0$ is a strictly lower triangular matrix, and $U_{k} \geq 0$ is a general matrix, and let $\left(B_{k}(\omega), C_{k}(\omega), E_{k}\right), k=1,2, \ldots, \ell$, be the SOR multisplitting of $M$. Let $\delta=\rho\left(D^{-1}(B+N)\right)$ and $\alpha=\max \left\{\delta, \max \left\{\rho\left(\left(B_{k}(\omega)\right)^{-1}\left|C_{k}(\omega)\right|\right) \mid 1 \leq k \leq \ell\right\}\right\}$. Let

$$
H_{\beta, \omega}=\sum_{k=1}^{\ell} E_{k}\left(\left(R_{\beta, \omega, k}\right)^{s}+\beta \sum_{j=0}^{s-1}\left(R_{\beta, \omega, k}\right)^{j}\left(B_{k}(\omega)\right)^{-1} N\right)
$$

be an iteration matrix of the relaxed two-stage multisplitting method using an outer splitting $A=M-N$ and inner splittings $M=B_{k}(\omega)-C_{k}(\omega)$, where $R_{\beta, \omega, k}=\beta\left(B_{k}(\omega)\right)^{-1} C_{k}(\omega)+(1-\beta) I$. Then the following hold.

(a) If $1<\omega<\frac{2}{1+\delta}$ and $0<\beta \leq 1$, then $\rho\left(H_{\beta, \omega}\right)<1$.

(b) If $\omega>0$ is chosen so that $\omega(1+\alpha)<2$ and if $0<\beta<\frac{2}{1+\omega \alpha+|1-\omega|}$, then $\rho\left(H_{\beta, \omega}\right)<1$.

\section{Numerical experiments}

In this section, we provide numerical experiments for the convergence of the relaxed two-stage multisplitting method using SOR multisplitting as inner splittings. All numerical values are computed using MATLAB. 
Example 4.1. Suppose that $\ell=3$. Consider an $H$-matrix $A$ of the form

$$
A=\left(\begin{array}{rrr}
F & I & 0 \\
-I & F & I \\
0 & -I & F
\end{array}\right), \quad F=\left(\begin{array}{rrr}
4 & -1 & 0 \\
1 & 4 & -1 \\
0 & 1 & 4
\end{array}\right), \quad I=\left(\begin{array}{lll}
1 & 0 & 0 \\
0 & 1 & 0 \\
0 & 0 & 1
\end{array}\right) \text {. }
$$

Let $A=M-N$, where

$$
M=\left(\begin{array}{ccc}
F & 0 & 0 \\
0 & F & 0 \\
0 & 0 & F
\end{array}\right), \quad N=\left(\begin{array}{rrr}
0 & -I & 0 \\
I & 0 & -I \\
0 & I & 0
\end{array}\right)
$$

Let $D=\operatorname{diag}(M), B=D-M$,

$$
\begin{gathered}
L_{11}=\left(\begin{array}{rrr}
0 & 0 & 0 \\
-1 & 0 & 0 \\
0 & 0 & 0
\end{array}\right), U_{11}=\left(\begin{array}{ccc}
0 & 1 & 0 \\
0 & 0 & 1 \\
0 & -1 & 0
\end{array}\right), \\
L_{12}=\left(\begin{array}{rrr}
0 & 0 & 0 \\
-1 & 0 & 0 \\
0 & -1 & 0
\end{array}\right), U_{12}=\left(\begin{array}{lll}
0 & 1 & 0 \\
0 & 0 & 1 \\
0 & 0 & 0
\end{array}\right), \\
L_{1}=\left(\begin{array}{ccc}
L_{11} & 0 & 0 \\
0 & L_{12} & 0 \\
0 & 0 & L_{12}
\end{array}\right), L_{2}=\left(\begin{array}{ccc}
L_{12} & 0 & 0 \\
0 & L_{11} & 0 \\
0 & 0 & L_{12}
\end{array}\right), L_{3}=\left(\begin{array}{ccc}
L_{12} & 0 & 0 \\
0 & L_{12} & 0 \\
0 & 0 & L_{11}
\end{array}\right), \\
U_{1}= \\
\left(\begin{array}{ccc}
U_{11} & 0 & 0 \\
0 & U_{12} & 0 \\
0 & 0 & U_{12}
\end{array}\right), U_{2}=\left(\begin{array}{ccc}
U_{12} & 0 & 0 \\
0 & U_{11} & 0 \\
0 & 0 & U_{12}
\end{array}\right), U_{3}=\left(\begin{array}{ccc}
U_{12} & 0 & 0 \\
0 & U_{12} & 0 \\
0 & 0 & U_{11}
\end{array}\right), \\
E_{1}=\left(\begin{array}{ccc}
I & 0 & 0 \\
0 & 0 & 0 \\
0 & 0 & 0
\end{array}\right), E_{2}=\left(\begin{array}{ccc}
0 & 0 \\
0 & I & 0 \\
0 & 0 & 0
\end{array}\right), E_{3}=\left(\begin{array}{ccc}
0 & 0 \\
0 & 0 & 0 \\
0 & 0 & I
\end{array}\right) .
\end{gathered}
$$

Then, $A=M-N$ is an $H$-compatible splitting of $A$ and $M=D-L_{k}-U_{k}$ is such that $\langle M\rangle=|D|-\left|L_{k}\right|-\left|U_{k}\right|$ for $k=1,2,3$. Let $\left(B_{k}(\omega), C_{k}(\omega), E_{k}\right)$, $k=1,2,3$, be the SOR multisplitting of $M$. That is, $B_{k}(\omega)=\frac{1}{\omega}\left(D-\omega L_{k}\right)$ and $C_{k}(\omega)=\frac{1}{\omega}\left((1-\omega) D+\omega U_{k}\right)$ for $k=1,2,3$. Then $\delta, \alpha$ and $H_{\beta, \omega}$ are defined as in Theorem 3.5. Note that $\delta=\rho\left(|D|^{-1}(|B|+|N|)\right) \approx 0.7071$ and $\frac{2}{1+\delta} \approx 1.1716$. For various values of $\omega$, the numerical values of $\alpha, \omega(1+\alpha)$ and $\frac{2}{1+\omega \alpha+|1-\omega|}$ are listed in Table 1 . Numerical values of $\rho\left(H_{\beta, \omega}\right)$ for various values of $\omega, \beta$ and $s$ are listed in Table 2 .

We next consider the more general case where $A$ is a large sparse blocktridiagonal $H$-matrix which is usually constructed from five-point finite difference discretization of the elliptic second order partial differential equations. For simplicity of exposition, suppose that $\ell=3$. Then $A$ can be partitioned into an $\ell \times \ell$ block-tridiagonal matrix of the form

$$
A=\left(\begin{array}{ccc}
A_{11} & A_{12} & 0 \\
A_{21} & A_{22} & A_{23} \\
0 & A_{32} & A_{33}
\end{array}\right)
$$


where $A_{i i}$ is a square matrix for $i=1,2,3$. Let $A=M-N$, where

$$
M=\left(\begin{array}{ccc}
A_{11} & 0 & 0 \\
0 & A_{22} & 0 \\
0 & 0 & A_{33}
\end{array}\right), \quad N=\left(\begin{array}{ccc}
0 & -A_{12} & 0 \\
-A_{21} & 0 & -A_{23} \\
0 & -A_{32} & 0
\end{array}\right)
$$

Let $A_{i i}=D_{i i}-L_{i i}-U_{i i}$ for $i=1,2,3$, where $D_{i i}=\operatorname{diag}\left(A_{i i}\right), L_{i i}$ is a strictly lower triangular matrix and $U_{i i}$ is a strictly upper triangular matrix. Let

$$
\begin{gathered}
L_{1}=\left(\begin{array}{ccc}
0 & 0 & 0 \\
0 & L_{22} & 0 \\
0 & 0 & L_{33}
\end{array}\right), L_{2}=\left(\begin{array}{ccc}
L_{11} & 0 & 0 \\
0 & 0 & 0 \\
0 & 0 & L_{33}
\end{array}\right), L_{3}=\left(\begin{array}{ccc}
L_{11} & 0 & 0 \\
0 & L_{22} & 0 \\
0 & 0 & 0
\end{array}\right), \\
U_{1}=\left(\begin{array}{ccc}
L_{11}+U_{11} & 0 & 0 \\
0 & U_{22} & 0 \\
0 & 0 & U_{33}
\end{array}\right), U_{2}=\left(\begin{array}{ccc}
U_{11} & 0 & 0 \\
0 & L_{22}+U_{22} & 0 \\
0 & 0 & U_{33}
\end{array}\right), \\
U_{3}=\left(\begin{array}{ccc}
U_{11} & 0 & 0 \\
0 & U_{22} & 0 \\
0 & 0 & L_{33}+U_{33}
\end{array}\right), D=\left(\begin{array}{ccc}
D_{11} & 0 & 0 \\
0 & D_{22} & 0 \\
0 & 0 & D_{33}
\end{array}\right), \\
E_{1}=\left(\begin{array}{ccc}
I & 0 & 0 \\
0 & 0 & 0 \\
0 & 0 & 0
\end{array}\right), E_{2}=\left(\begin{array}{lll}
0 & 0 & 0 \\
0 & I & 0 \\
0 & 0 & 0
\end{array}\right), E_{3}=\left(\begin{array}{ccc}
0 & 0 & 0 \\
0 & 0 & 0 \\
0 & 0 & I
\end{array}\right) .
\end{gathered}
$$

Then, $A=M-N$ is an $H$-compatible splitting of $A$ and $M=D-L_{k}-U_{k}$ is such that $\langle M\rangle=|D|-\left|L_{k}\right|-\left|U_{k}\right|$ for $k=1,2,3$. Let $\left(B_{k}(\omega), C_{k}(\omega), E_{k}\right)$, $k=1,2,3$, be the SOR multisplitting of $M$. That is, $B_{k}(\omega)=\frac{1}{\omega}\left(D-\omega L_{k}\right)$ and $C_{k}(\omega)=\frac{1}{\omega}\left((1-\omega) D+\omega U_{k}\right)$ for $k=1,2,3$. Using the ideas and techniques mentioned above, we provide numerical results for the following example.

Example 4.2. Consider the following Poisson PDE

$$
\left\{\begin{array}{lll}
-u_{x x}-u_{y y} & =g & \text { in } \Omega \\
u(x, y) & =0 & \text { on } \partial \Omega,
\end{array}\right.
$$

where $\Omega=(0,1) \times(0,1)$ and $\partial \Omega$ denotes the boundary of $\Omega$. The five-point finite difference discretization for the PDE (20) is used. We have used a uniform mesh of $\Delta x=\Delta y=1 / 46$, which lead to a block-tridiagonal $H$-matrix $A$ of order $n=45^{2}=2025$, where $\Delta x$ and $\Delta y$ refer to the mesh sizes in the $x$-direction and $y$-direction, respectively. $\delta, \alpha$ and $H_{\beta, \omega}$ are defined as in Theorem 3.5. Note that $\delta=\rho\left(|D|^{-1}(|B|+|N|)\right) \approx 0.9977$ and $\frac{2}{1+\delta} \approx 1.0012$ for this example. For various values of $\omega$, the numerical values of $\alpha, \omega(1+\alpha)$ and $\frac{2}{1+\omega \alpha+|1-\omega|}$ are listed in Table 3 . Numerical values of $\rho\left(H_{\beta, \omega}\right)$ for various values of $\omega, \beta$ and $s$ are listed in Table 4.

For test problems used in this paper, the upper bound of $\beta$, which is

$$
\frac{2}{1+\omega \alpha+|1-\omega|}
$$


becomes maximum when $\omega=1$ (see Tables 1 and 3). All numerical results are consistent with the theoretical results provided in this paper (see Tables 1 to 4). From Tables 2 and 4, it may be concluded that the optimal pairs of $\beta$ and $\omega$ for which $\rho\left(H_{\beta, \omega}\right)$ is minimized vary depending upon $s$ and the problem to be considered.

TABLE 1. Numerical values of $\alpha, \omega(1+\alpha)$ and $\frac{2}{1+\omega \alpha+|1-\omega|}$ for Example 4.1.

\begin{tabular}{|cccc|}
\hline$\omega$ & $\alpha$ & $\omega(1+\alpha)$ & $\frac{2}{1+\omega \alpha+|1-\omega|}$ \\
\hline 0.2 & 0.8683 & 0.3737 & 1.0133 \\
0.3 & 0.8006 & 0.5402 & 1.0308 \\
0.4 & 0.7316 & 0.6926 & 1.0567 \\
0.5 & 0.7071 & 0.8536 & 1.0790 \\
0.8 & 0.7071 & 1.3657 & 1.1327 \\
0.9 & 0.7071 & 1.5364 & 1.1518 \\
1.0 & 0.7071 & 1.7071 & 1.1716 \\
1.1 & 0.7071 & 1.8778 & 1.0651 \\
1.15 & 0.7071 & 1.9632 & 1.0188 \\
1.17 & 0.7071 & 1.9973 & 1.0013 \\
1.18 & 0.7071 & 2.0144 & 0.9929 \\
\hline
\end{tabular}

TABLE 2. Numerical values of $\rho\left(H_{\beta, \omega}\right)$ for Example 4.1 .

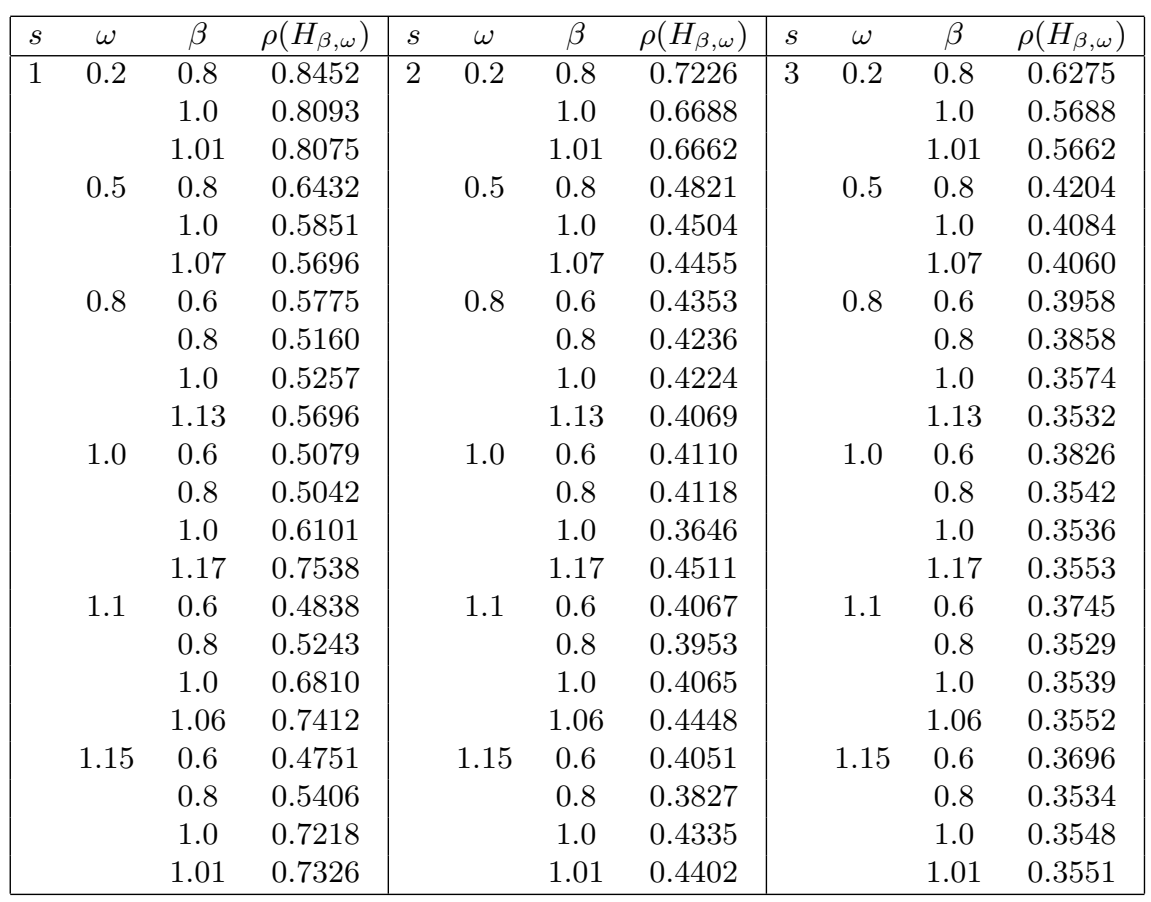


TABLE 3. Numerical values of $\alpha, \omega(1+\alpha)$ and $\frac{2}{1+\omega \alpha+|1-\omega|}$ for Example 4.2.

\begin{tabular}{|cccc|}
\hline$\omega$ & $\alpha$ & $\omega(1+\alpha)$ & $\frac{2}{1+\omega \alpha+|1-\omega|}$ \\
\hline 0.2 & 0.9978 & 0.3996 & 1.0002 \\
0.5 & 0.9977 & 0.9988 & 1.0006 \\
0.8 & 0.9977 & 1.5981 & 1.0009 \\
1.0 & 0.9977 & 1.9977 & 1.0012 \\
1.001 & 0.9977 & 1.9997 & 1.0002 \\
1.002 & 0.9977 & 2.0017 & 0.9992 \\
\hline
\end{tabular}

TABLE 4. Numerical values of $\rho\left(H_{\beta, \omega}\right)$ for Example 4.2 .

\begin{tabular}{|c|c|c|c|c|c|c|c|c|c|c|c|}
\hline$s$ & $\omega$ & $\beta$ & $\rho\left(H_{\beta, \omega}\right)$ & $s$ & $\omega$ & $\beta$ & $\rho\left(H_{\beta, \omega}\right)$ & $s$ & $\omega$ & $\beta$ & $\rho\left(H_{\beta, \omega}\right)$ \\
\hline \multirow[t]{18}{*}{1} & 0.2 & 0.8 & 0.9996 & 2 & 0.2 & 0.8 & 0.9993 & 3 & 0.2 & 0.8 & 0.9989 \\
\hline & & 1.0 & 0.9995 & & & 1.0 & 0.9991 & & & 1.0 & 0.9986 \\
\hline & & 1.0001 & 0.9995 & & & 1.0001 & 0.9991 & & & 1.0001 & 0.9986 \\
\hline & 0.5 & 0.8 & 0.9991 & & 0.5 & 0.8 & 0.9981 & & 0.5 & 0.8 & 0.9972 \\
\hline & & 1.0 & 0.9988 & & & 1.0 & 0.9977 & & & 1.0 & 0.9966 \\
\hline & & 1.0005 & 0.9988 & & & 1.0005 & 0.9977 & & & 1.0005 & 0.9966 \\
\hline & 0.8 & 0.6 & 0.9989 & & 0.8 & 0.6 & 0.9978 & & 0.8 & 0.6 & 0.9967 \\
\hline & & 0.8 & 0.9985 & & & 0.8 & 0.9971 & & & 0.8 & 0.9956 \\
\hline & & 1.0 & 0.9981 & & & 1.0 & 0.9963 & & & 1.0 & 0.9946 \\
\hline & & 1.0008 & 0.9981 & & & 1.0008 & 0.9963 & & & 1.0008 & 0.9946 \\
\hline & 1.0 & 0.6 & 0.9986 & & 1.0 & 0.6 & 0.9972 & & 1.0 & 0.6 & 0.9959 \\
\hline & & 0.8 & 0.9981 & & & 0.8 & 0.9963 & & & 0.8 & 0.9946 \\
\hline & & 1.0 & 0.9977 & & & 1.0 & 0.9954 & & & 1.0 & 0.9933 \\
\hline & & 1.0011 & 0.9999 & & & 1.0011 & 0.9954 & & & 1.0011 & 0.9933 \\
\hline & 1.001 & 0.6 & 0.9986 & & 1.001 & 0.6 & 0.9972 & & 1.001 & 0.6 & 0.9959 \\
\hline & & 0.8 & 0.9981 & & & 0.8 & 0.9963 & & & 0.8 & 0.9946 \\
\hline & & 1.0 & 0.9997 & & & 1.0 & 0.9954 & & & 1.0 & 0.9933 \\
\hline & & 1.0001 & 0.9999 & & & 1.0001 & 0.9954 & & & 1.0001 & 0.9933 \\
\hline
\end{tabular}

Acknowledgements. The author would like to thank the anonymous referee for useful comments and constructive suggestions which substantially improved the quality of this paper.

\section{References}

[1] A. Berman and R. J. Plemmons, Nonnegative Matrices in the Mathematical Sciences, Computer Science and Applied Mathematics. Academic Press [Harcourt Brace Jovanovich, Publishers], New York-London, 1979.

[2] R. Bru, V. Migallón, J. Penadés, and D. B. Szyld, Parallel, synchronous and asynchronous two-stage multisplitting methods, Electron. Trans. Numer. Anal. 3 (1995), 24-38.

[3] A. Frommer and G. Mayer, Convergence of relaxed parallel multisplitting methods, Linear Algebra Appl. 119 (1989), 141-152.

[4] M. Neumann and R. J. Plemmons, Convergence of parallel multisplitting iterative methods for M-matrices, Linear Algebra Appl. 88/89 (1987), 559-573.

[5] D. P. O'Leary and R. E. White, Multisplittings of matrices and parallel solution of linear systems, SIAM J. Algebraic Discrete Methods 6 (1985), no. 4, 630-640.

[6] D. B. Szyld and M. T. Jones, Two-stage and multisplitting methods for the parallel solution of linear systems, SIAM J. Matrix Anal. Appl. 13 (1992), no. 2, 671-679.

[7] R. S. Varga, Matrix Iterative Analysis, Prentice-Hall, Inc., Englewood Cliffs, N.J. 1962

[8] D. Wang, On the convergence of the parallel multisplitting AOR algorithm, Linear Algebra Appl. 154/156 (1991), 473-486. 
[9] J. H. Yun, Performance of ILU factorization preconditioners based on multisplittings, Numer. Math. 95 (2003), no. 4, 761-779.

Department of Mathematics

College of Natural Sciences

Chungbuk National University

Cheonguu 361-763, Korea

E-mail address: gmjae@chungbuk.ac.kr 